\title{
Protection against Plasmodium falciparum malaria in chimpanzees by immunization with the conserved pre- erythrocytic liver-stage antigen 3
}

\author{
Pierre Daubersies ${ }^{1}$, Alan W. Thomas ${ }^{2}$, Pascal Millet ${ }^{3}$, Karima Brahimi ${ }^{1}$, Jan A.M. \\ Langermans ${ }^{2}$, Benjamin Ollomo ${ }^{3}$, Lbachir Ben Mohamed ${ }^{1}$, Bas Slierendregt ${ }^{2}$, Wijnand \\ Eling ${ }^{4}$, Alex Van Belkum ${ }^{2}$, Guy Dubreuil ${ }^{3}$, Jacques F.G.M. Meis ${ }^{5}$, Claudine Guérin- \\ Marchand $^{1}$, Sylvie Cayphas ${ }^{6}$, Joe Cohen ${ }^{6}$, Hélène Gras-Masse ${ }^{7} \&$ Pierre Druilhe ${ }^{1}$ \\ ${ }^{1}$ Unité de Parasitologie Biomédicale, Institut Pasteur, 28 rue du Dr Roux, 75015 Paris, France \\ ${ }^{2}$ Biomedical Primate Research Centre, Department of Parasitology, Postbox 3306, 2280 GH Rijswijk, The Netherlands \\ ${ }^{3}$ Centre International de Recherches M édicales de Franceville, B.P.769, Franceville, Gabon \\ ${ }^{4}$ D epartment of M edical Microbiology and ${ }^{5}$ Department of M edical Parasitology, \\ University of Nijmegen, P.O. Box 9101, Nijmegen, The Netherlands, \\ ${ }^{6}$ SmithKline Beecham Biologicals S.A., rue de I'Institut 89, B-1330 Rixensart, Belgium \\ 7Laboratoire de Chimie des Bio-M olécules, URA CNRS 1309, Institut Pasteur, 1 rue du Pr Calmette, BP 245, 59019 \\ Lille Cedex, France P.M. present address: Centre René Labusquière, Université Victor Segalen, \\ Bordeaux II, 146 rue Léo-Saignat, 33076 Bordeaux Cedex, France \\ C.G.-M. present address: Unité d'Immunoallergologie, Institut Pasteur, 28 rue du Dr Roux, 75015 Paris, France \\ Correspondence should be addressed to P.D.; Email: druilhe@pasteur.fr
}

\begin{abstract}
In humans, sterile immunity against malaria can be consistently induced through exposure to the bites of thousands of irradiated infected mosquitoes. The same level of protection has yet to be achieved using subunit vaccines. Recent studies have indicated an essential function for intrahepatic parasites, the stage after the mosquito bite, and thus for antigens expressed during this stage. We report here the identification of liver-stage antigen 3, which is expressed both in the mosquito and liver-stage parasites. This Plasmodium falciparum 200-kilodalton protein is highly conserved, and showed promising antigenic and immunogenic properties. In chimpanzees (Pan troglodytes), the primates most closely related to humans and that share a similar susceptibility to $P$. falciparum liver-stage infection, immunization with LSA-3 induced protection against successive heterologous challenges with large numbers of $P$. falciparum sporozoites.
\end{abstract}

Irradiated sporozoite vaccines remain experimental because sporozoites, the parasite present in mosquito salivary glands, cannot be artificially cultured and the only effective means of administration is through 12-14 exposures to hundreds of infected mosquito bites ${ }^{1,2}$. Current sporozoite candidate vaccines have not yet achieved similar protection ${ }^{3-5}$, and their use is hampered by substantial polymorphism in immunologically important regions and by low immunogenicity ${ }^{3}$. The basis of the strong immunological protection induced in humans by vaccination with radiation-attenuated pre-erythrocytic malaria parasites is poorly understood. However, there is now an increasing body of evidence that the transformation of the irradiated sporozoites into live but developmentally arrested intrahepatic liver trophozoites, which occurs at low (15-20 krad) irradiation doses but not at high (23-30 krad) irradiation doses ${ }^{6}$, is required to induce protection ${ }^{7,8}$. We therefore focused our efforts on the characterization of antigens expressed during this incompletely elucidated phase of the Plasmodium life cycle $e^{9}$.

\section{Selection of liver-stage antigen 3 by human responses}

The different responses of hosts immunized with irradiated sporozoites could provide a screening strategy for molecules relevant to protection. Thus, we screened 120 phage lambda clones previously identified as expressing $\mathrm{P}$. falciparum polypeptides that are expressed during pre-erythrocytic stage parasite development $^{9,10}$ and that are derived from about 20 distinct genes ${ }^{9-12}$. We screened a clone corresponding to each of these putative genes using eight serum samples from human volunteers (four of six protected) and from chimpanzees (one of two protected) immunized with sporozoites irradiated at low or high doses. A single clone (DG729) reacted only with sera from protected humans and chimpanzees. After confirming this differential reactivity further with a peptide derived from this fragment (Table 1), we used this clone for further investigation.

\section{Characterization of liver-stage antigen 3}

We used DG729 to probe a genomic library of P. falciparum (K1 strain). One clone contained the whole gene corresponding to DG729; we called it liver-stage antigen 3 (LSA-3) and determined its sequence, expression, location and conservation (Figs. 1-3 and http://www.pasteur.fr/parmed/Isa3). We identified a singlecopy gene consisting of a 'mini-exon' 1 (198 base pairs), a 'miniintron' (168 base pairs) and a large exon 2 (5.16 kilobases), a structure similar to that of other surface antigens of $P$. falciparum $^{13}$. The gene for LSA-3 was located on chromosome 2 (ref. 14), where it was called ring-infected erythrocyte surface antigen 


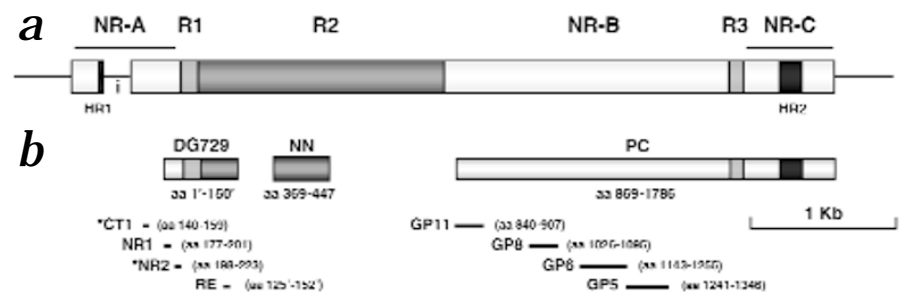

(RESA)-H3 (Genbank accession number, AE001424). LSA-3, with a predicted molecular weight of $200 \mathrm{kDa}$ (in K1), is made up of large non-repeated sequences flanking three Glu-rich repeated regions, a feature that extends the known P. falciparum Glu-rich antigen network ${ }^{15}$ to include a pre-erythrocytic component. We determined the location of the original fragment (DG729) and of the peptides corresponding to repeat region $\mathrm{R} 2$ and non-repetitive regions NR-A and NR-B (Fig. 1b). Natural or artificially induced antibodies against the non-repeated peptides and the recombinant protein gl utathione S-transferase (GST)-PC (see fig. 1) were not cross-reactive with the repeated Glu-rich regions, and we used these for further studies.

We confirmed preerythrocytic expression of LSA-3 (Figs. 2 and 3 and http://www.pasteur.fr/parmed/lsa3) by RT-PCR (primers $i 1$ and $i 2$ ) of total RNA and western blot analysis of protein extracts, isolated in both cases from sporozoites, and also by immunofluorescence antibody test (IFAT) of infected liver sections and dry or wet sporozoite preparations, using antibodies against a non-cross-reactive portion of the protein. In 5- and 6day-old liver schizonts, LSA-3 was present in the parasitophorous vacuole and at the periphery of maturing hepatic merozoites. This location is consistent with the molecular structure of this protein, which has two hydrophobic regions (Fig. 1a). We were unable to detect mRNA of LSA-3 by northern blot analysis of RNA from erythrocytic stages. Western blot analysis and IFAT of infected red blood cells were al so consistently negative with non-cross-reactive antibodies. However, we obtained reactivity using antibodies against the Glu-rich repeat region (http://www.pasteur.fr/parmed/lsa3), which reflects observations of a putatively homologous antigen (D260) in intra-erythrocytic parasites identified solely using such antibodies ${ }^{16}$.

Given concerns about the polymorphism of many malaria vaccine candidate molecules ${ }^{3,17,18}$, we investigated naturally occurring sequence variation in LSA-3 (http://www.pasteur.fr/parmed/lsa3). We consistently detected the gene by PCR amplification of the NR-A region (primers S1 and S2) in a total of $111 \mathrm{P}$. falciparum isolates, strains or clones of various geographical origins. Using LSA-3-specific antibodies with IFAT, we also detected expression of LSA-3 in liver schizonts of two distinct strains and in all the sporozoites from 30 wild isolates that developed in mosquitoes fed in vitro on Thai gametocytes. The repeat regions R1 and R3 were highly conserved, but there was variation in the number and order of the repeat units of R2 among different parasite lines. However, this did not affect the predicted conserved $\alpha$-helical organization, a secondary structure considered important in defining principal B-cell epitopes, as antibodies that recognize R2 did indeed produce positive results by IFAT with all the parasites tested. The non-repeated portions of exon 2, where many T-hel per and cytotoxic T-Iymphocyte epitopes are found ${ }^{19-21}$, had a considerable degree of amino-acid sequence conservation between different
Fig. 1 Gene for LSA-3, recombinant proteins and peptides. a, NR-A, NR-B and NR-C, non-repeated sequences; R1-R3, three repeat blocks; $\mathrm{HR} 1$ and HR2, hydrophobic regions possibly corresponding to the N-terminal signal peptide and the anchor region, respectively; intron (i). b, Location of the sequences encoding for LSA-3 in the recombinant fusion proteins (first line) and the synthetic peptides (bars) used here (aminoacid numbering, http://www.pasteur.fr/parmed/lsa3. aa, amino acids; *, palmitoyl-conjugated lipopeptides; ' , amino acid positions in T9/96 clone.

parasites (more than $95.5 \%$ homology). The sequence of the non-repetitive (NR) 2 (see Fig.1) peptide was fully conserved among $\mathrm{K} 1$ and T9/96 parasites (the source of the immunizing proteins), the NF54 parasites used for sporozoite challenges and $27 \mathrm{P}$. falciparum samples of various geographical origins ${ }^{19}$. An HLA-B53-restricted epitope identified in the NR-B region of LSA3 (present in GST-PC recombinant protein) was also free of variation in clone 3D7 and in 18 Gambian isolates ${ }^{21}$. This conservation of immunologically important epitopes contrasts with substantial polymorphism in current pre-erythrocytic vaccine candidates.

\section{Validation of the chimpanzee for vaccine studies}

The chimpanzee is the primate most closely related to humans genetically (98.4\% homology at the DNA level $)^{22}$. We used many preliminary stringent tests in control chimpanzees to confirm the suitability of this model for vaccine evaluation. As cost and ethical considerations precluded the use of large numbers of animals, high reproducibility of the infection in this model system was essential. In a prel iminary experiment (Table 2, group I), we confirmed that in the chimpanzee, protection by immunization with irradiated sporozoite was radiation dose-dependent, as it is in humans, and we confirmed the use of detection of the infected red blood cells as an assay of protection. The results allowed us to define many important parameters. Chimpanzees developed a powerful protective response after immunization with irradiated sporozoite, as in humans. Moreover, chimpanzees, like humans, were also susceptible to successive challenges, in contrast to lower primates or rodents, which become refractory after the first challenge $e^{23}$. In two chimpanzees, five

Table 1 Differential reactivity of sera from protected or nonprotected humans or chimpanzees with peptide NR2

\begin{tabular}{lcccc}
$\begin{array}{l}\text { Code or } \\
\text { Name }\end{array}$ & $\begin{array}{c}\text { Sporozoite } \\
\text { irradiation dose }\end{array}$ & $\begin{array}{c}\text { IFAT titer on } \\
\text { sporozoite }\end{array}$ & $\begin{array}{c}\text { Protective } \\
\text { status }\end{array}$ & $\begin{array}{c}\text { NR2 peptide } \\
\text { (amino acids } \\
\text { 198-223) }\end{array}$ \\
V4 & 23.6 & 4,096 & not protected & 0.5 \\
V5 & 23.6 & 32,000 & 2-day delay & 0.5 \\
Japie & 30 & 32,000 & not protected & 0.7 \\
V6 & 20.8 & 5,120 & Protected & 3.8 \\
V7 & 20.8 & 41,960 & Protected & 2.6 \\
V8 & 20.8 & 40,960 & Protected & 4.8 \\
WR4 & 15 & 3,200 & Protected & 3.4 \\
Carl & 18 & 6,400 & Protected & 2.3 \\
\hline
\end{tabular}

Immunoglobulin G-specific antibodies against peptide NR2 were measured by enzymelinked immunoassay (in arbitrary units) in sera from human volunteers (codes) and chimpanzees (names, in italic) immunized with sporozoites irradiated at low or high dose (in krad). Codes, immunization schemes, sporozoite IFAT titers and protective status determination for human volunteers V4-V8 and WR4 have been described ${ }^{1,2}$. Similar experiments with peptides NR1 and RE (Fig. 1) yielded negative results with these sera (data not shown). 
consecutive challenges (three medium-dose and two high-dose) over 2 years provided reproducible results. Furthermore, as a result of the high dose of inoculated sporozoites, we obtained positive blood smears reproducibly in non-protected chimpanzees on days 6 or 7. This corresponded to the first invasion of red cells by merozoites released from intrahepatocytic schizonts. Finally, in the non-splenectomized chimpanzee, erythrocytic infections normally remain sub-clinical and self-limiting, which in fact occurred despite the high-dose challenges. We confirmed these results in two more chimpanzees (Table 2, Emmanuel and Fauzi).

\section{LSA-3 induces protection in chimpanzees}

Having established the suitability of the chimpanzee for our use, we next assessed the protective value of LSA-3 immunization by challenge with viable $\mathrm{P}$. falciparum sporozoites. In preliminary experiments, we immunized two chimpanzees (Dirk and Ruud) with a mixture of LSA-3 and LSA-1 recombinant proteins. We obtained full protection against three challenges over several months only in the chimpanzee that responded to LSA-3 (Dirk), whereas both responded to LSA-1 (data not shown). In liver biopsies of Dirk on day 5, we detected only one liver schizont of 'unhealthy' appearance and infiltrated by leukocytes in the 300 liver sections screened (Fig. 3). In contrast, we found 2,500 and 750 hepatic schizonts of 'healthy' appearance in the two nonprotected control chimpanzees.

Thus, we focused further immunization and challenge experiments on LSA-3 alone. We used two groups of chimpanzees to

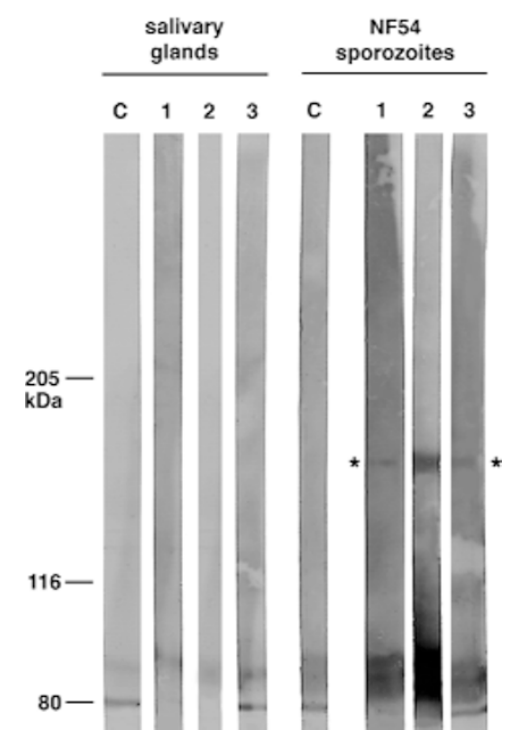

Fig. 2 LSA-3 expression in $P$. falciparum sporozoites. Western blot analysis of protein extracts from NF54 sporozoites and control uninfected mosquito salivary glands using mouse antisera against control GST (C), GST-PC (lane 1), peptides GP5, GP6, GP8 or GP11 (lane 2) or GST-729 (lane 3). *, LSA-3 (175-kDa protein, in agreement with the theoretical molecular weight of LSA-3 in this parasite strain). Left margin, molecular sizes.

evaluate lipopeptide and recombinant protein formulations (Table 2, groups II-III). In group II, one chimpanzee (Gerda) was initially immunized solely with the NR2 lipopeptide of LSA-3, and was boosted by recombinant LSA-3 molecules in Montanide ISA 51 adjuvent (SEPPIC, France). Gerda was fully protected when challenged with $1 \times 10^{7}$ sporozoites, whereas the control chimpanzee receiving Montanide ISA 51 alone was not (Fig. 4a).

Table 2: Results of challenge experiments in chimpanzees

\begin{tabular}{|c|c|c|c|c|c|}
\hline \multicolumn{2}{|c|}{ Animal Groups } & \multirow{2}{*}{$\begin{array}{l}\text { Immunization } \\
\text { dates } \\
\text { (weeks) }\end{array}$} & \multirow{2}{*}{$\begin{array}{l}\text { Challenge } \\
\text { dates } \\
\text { (weeks) }\end{array}$} & \multicolumn{2}{|c|}{ Protection } \\
\hline Chimpanzee & Immunization protocols & & & $\begin{array}{l}\text { low-dose } \\
2 \times 10^{4}\end{array}$ & $\begin{array}{l}\text { high-dose } \\
10^{7}\end{array}$ \\
\hline & Group $1^{a}$ & $0,8,24,44,65$ & 93,123 & & \\
\hline Carl & 18 krad-irradiated sporozoites & & & + & + \\
\hline Japie & 30-krad-irradiated sporozoites & & & - & - \\
\hline Marcel & unimmunised control & & & - & - \\
\hline Theo & unimmunised control & & & - & - \\
\hline Gerda & $\begin{array}{l}\text { Group II } \\
\text { (lipopep. NR2) then GST-rec in ISA51 }\end{array}$ & $\begin{array}{l}0,3,13,31 \\
\text { then }\end{array}$ & 60 & nd & + \\
\hline Lianne & control ISA 51 & $40,45,48,50$ & & nd & - \\
\hline & Group III & $0,3,6$ & $21,29^{b}$ & & \\
\hline Mopia & (lipo)pep. & & & + & $\mathrm{d} 2$ \\
\hline Mgbado & (lipo)pep. & & & + & $\mathrm{d} 2$ \\
\hline Judy & GST-rec/microbeads & & & $\mathrm{d} 1$ & - \\
\hline Ondele & control GST/microbeads & & & - & - \\
\hline Makata & unimmunized control & & & - & - \\
\hline & Group IV & $0,4,8$ & 13 & & \\
\hline Patty & (lipo)pep. ${ }^{c}$ & & & + & nd \\
\hline Wendy & GST-rec in SBAS2 & & & + & nd \\
\hline Willy & GST-rec in SBAS2 & & & - & nd \\
\hline Helen & control SBAS2 & & & - & nd \\
\hline & Group V & & 33,46 & & \\
\hline Cindy & GST-rec in SBAS2 & $0,4,8,26^{d}$ & & + & + \\
\hline Marty & GST-rec in SBAS2 & & & $\mathrm{d} 1$ & - \\
\hline Emmanuel & 18 krad-irradiated sporozoites & $5,8,11,26^{\mathrm{e}}$ & & + & + \\
\hline Fauzi & 30 krad-irradiated sporozoites & & & - & - \\
\hline
\end{tabular}

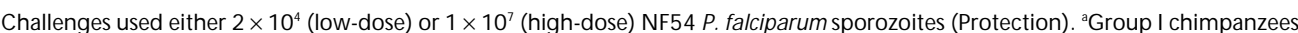
received three additional challenges (two low-dose and one high-dose), which led each time to similar results; that is, a reproducible protection only in Carl (data not shown). ${ }^{b} \mathrm{High}$-dose challenge with $5 \times 10^{6}$ sporozoites. 'Same mixture as in group III but without peptide $\mathrm{CT} 1$. ${ }^{\mathrm{d}} \mathrm{Cindy}$ and Marty. ${ }^{\mathrm{F}}$ Fauzi. + , complete protection; $\mathrm{d} 1$ and $\mathrm{d} 2$ : 1 -day (d1) and 2-day (d2) delay to patency compared with that of controls; nd, not done; lipopep., lipopeptide; (lipo)pep., mixture of NR1, NR2 and CT1 lipopeptides and RE peptide; GST-rec, mixture of recombinants GST-DG729, GST-NN and GST-PC.
In Gerda, boosting with the recombinant LSA-3 formulation did not induce any detectable increase in the strong B-cell, T helper-cell and cytotoxic T-lymphocyte responses already evoked by the initial lipopeptide-peptide injections ${ }^{19,20}$. Therefore, we sought to determine whether the simple and well-tolerated peptide formulation alone could induce protection. We immunized two chimpanzees, Mopia and Mgbado, with LSA-3 lipopeptide-peptides alone (Table 2, group III). We obtained protection against a first challenge with $2 \times 10^{4}$ sporozoites in both. Experiments with the same group included an investigation of the effects of microbead presentation of recombinant proteins without adjuvant in one chimpanzee (Judy), which resulted in a 1-day delay to 'patency' (time of blood infection after sporozoite challenge) (Fig. 4b). After a subsequent highdose sporozoite challenge $(5 \times$ $10^{6}$ sporozoites), both Mopia and Mgbado demonstrated a distinct 2-day delay to patency 

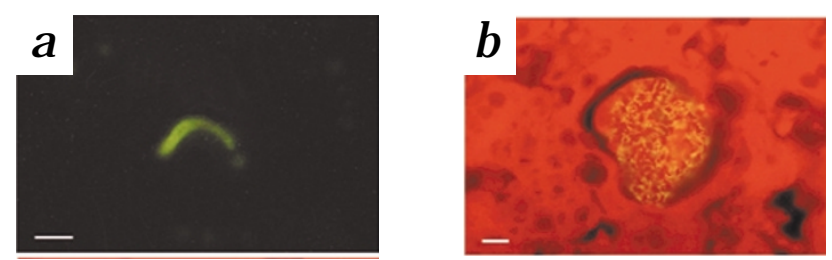

Fig. 3 Immunostaining of P. falciparum pre-erythrocytic stages with antibodies against LSA-3. a, Sporozoites stained by IFAT with human antibodies affinity-purified on recombinant bgal-DG729. b. Staining by IFAT of P. falciparum liver stages on day 6 after challenge32 from a chimpanzee, using the antibodies induced by lipopeptide NR2 injection19 in chimpanzee Gerda (additional pictures,http://www.pasteur.fr/parmed//sa3). c and d, The sin-
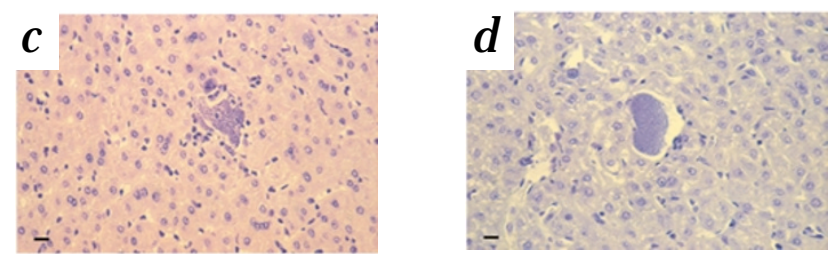

gle residual liver schizont detected in chimpanzee Dirk (day 5 after challenge) seems infiltrated by lymphomononuclear cells (c, compared with one of the many healthy schizonts in control chimpanzee Peer (d) (total of about 2,500 schizonts per 300 liver sections). Scale bars represent $5 \mathrm{~mm}$ (a) and $20 \mathrm{~mm}(\mathrm{~b}-\mathrm{d})$.

panzee immune responses can be made and legitimately compared with those of humans ${ }^{19,20}$. The fact that parasitological and immunological events can be directly examined in liver biopsies, a possibility excluded for infected humans, is of considerable relevance.

We immunized nine chimpanzees using lipopeptides in saline or polypeptides in either M ontanide or SBAS2. Full sterile protection was induced in six of these nine chimpanzees on first challenge. After taking into consideration the substantial delay compared with that of controls, which corresponds to a considerable decrease in liver parasite load ${ }^{24}$, we were able to extend the protective effect induced by LSA-3 to eight of the nine chimpanzees. We obtained protection in 11 of the 14 challenges, which were done in chimpanzees immunized with LSA-3. This protection was complete for 7 of the 11 challenges. All the control chimpanzees here showed a consistent pattern in the early appearance and the course of the blood-stage parasitemia after each of the 12 challenges with viable parasites. Demonstration of this reproducibility in controls is essential in the interpretation of our data. After taking into consideration other groups of chimpanzees used in preliminary experiments in which complex schemes of immunization or other pre-erythrocytic antigens were tested (data not shown), we were able to confirm the reproducibility of the model with an additional 26 successful challenges.

A chief concern of vaccine candidates at present is genetic polymorphism ${ }^{17,18}$. It is therefore encouraging that protection was induced against a heterologous challenge (NF54 strain) in outbred chimpanzees immunized with LSA-3 molecules whose sequences were derived from K1 and T9/96 parasites. An advantage of LSA-3 may reside in its limited sequence polymorphism. a

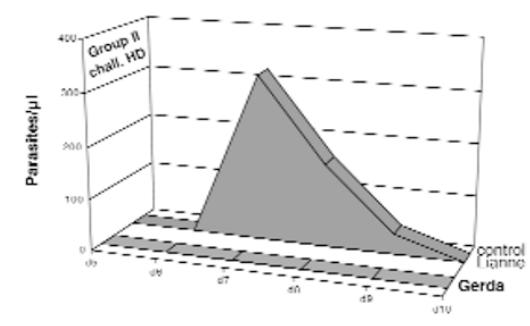

b

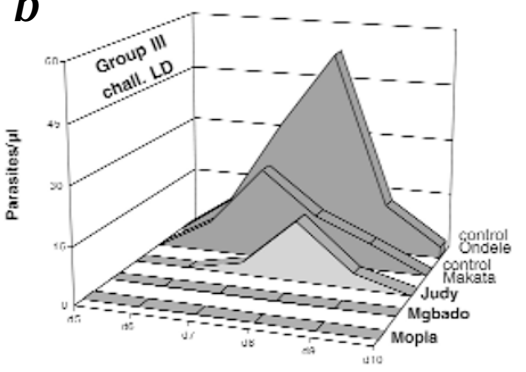

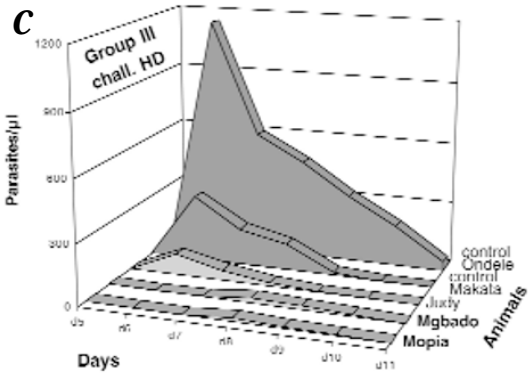

Fig. 4 Blood parasitemia courses in groups II and III. Chimpanzees from group II (a) and group III (b and c) after high-dose (HD) or low-dose (LD) challenge (chall.) with NF54 sporozoites. Bold names, totally or partially protected chimpanzees. Parasitemia scales are different for each challenge, as expected from challenges with different numbers of sporozoites. The day of patency in control and non-protected chimpanzees is the same for a given challenge inoculum within each group (here and in other groups not shown here). 
We investigated a variety of immunization strategies in the course of this work. Our data confirm the utility of SBAS2. The results we obtained with simple peptide and lipopeptide formulations are also particularly encouraging, as these vaccines do not require adjuvant and are relatively easy to produce with Good M anufacturing Practice conditions ${ }^{25}$. We detected no local or general reactions in our chimpanzees after lipopeptide injections, an observation consistent with previous experience with similar formulations derived from simian immunodeficiency virus in macaques ${ }^{26}$ and hepatitis B surface antigen ${ }^{27}$ or human immunodeficiency virus ${ }^{25}$ in humans. This bodes well for future clinical trials.

\section{Methods}

Selection of clone DG729. Dot-blot analysis of the $\beta$-galactosidase-fused recombinant proteins encoded by the pre-erythrocytic clones was done on nitrocellulose as described ${ }^{9}$, using human and chimpanzee sera diluted 1:100. Enzyme-linked immunoassays were done in duplicate as described ${ }^{28}$ on sera diluted 1:100, using coating solutions of $0.3,3$ and $10 \mu \mathrm{g} / \mathrm{ml} \mathrm{NR1}$, NR2 and (RE) (see Fig. 1)peptides, respectively, in PBS. Enzyme-linked immunoassay titers are expressed in arbitrary units representing the ratio of the mean absorbance values from test sera to the mean absorbance value plus three standard deviations from 10 control samples assayed in parallel in the same plate. Results were considered positive for ratios above one (ref. 19).

LSA-3 cloning and characterization. Detailed description of molecular methods, gene cloning, sequence data, protein characteristics and description of the recombinant proteins and of the peptides are available at http://www.pasteur.fr/parmed/Isa3. The primers used for PCR were S1 (nucleotides 161-184) and S2 (nucleotides 454-432), and those for RT-PCR were i1 (nucleotides 695-722) and i2 (nucleotides 824-799); numbering refers to the sequence of the gene for LSA-3 sequence of K1 (Genbank accession number, AJ007010). All mouse sera used for the western blot (at a dilution of 1:100) in Fig. 2 were obtained after three subcutaneous injections of $100 \mathrm{mg}$ immunogen emulsified in SBAS2 (ref. 4). Long synthetic peptides GP5, GP6, GP8 and GP11 (see Fig. 1) were synthesized as described29 (Fig. 1, position).

Immunogens injected in chimpanzees. Sequences of the immunogens consisted of clone DG729 and inserts NN (see Fig. 1) and PC, as well as peptides NR1, NR2, RE and CT1 (Fig. 1, location; details available at http://www.pasteur.fr/parmed/Isa3). Clone DG729, as well as inserts NN and PC were expressed as GST-fused recombinants and were purified according to the manufacturer's recommendations (Invitrogen, Carlsbad, California). Recombinant proteins GST-DG729, GST-NN and GST-PC were designed to cover $95 \%$ of the LSA-3 antigen and were used as a mixture (called LSA-3 GST-rec). Peptides NR1, NR2 and CT1 were also synthesized as palmitoyl-conjugated lipopeptides (* in Fig. 1), as described19. The combination of synthetic compounds consisted of a mixture of NR1, NR2 and CT1 lipopeptides and of RE peptide. All peptides and lipopeptides were purified to a purity of more than $90 \%$ by reverse-phase chromatography, and the impurities consisted essentially of related peptides of shorter sequences19.

Chimpanzee immunizations and challenges. None of the chimpanzees here had been exposed to malaria infections or malarial antigens previously. Recombinant and synthetic compounds were injected subcutaneously, at a dose of $100 \mu \mathrm{g}$ for each peptide and/or lipopeptides, and/or $50 \mu \mathrm{g}$ for each protein. Lipopeptides were always injected in PBS and, except where described otherwise, peptides and recombinants were emulsified in Montanide ISA51. Chimpanzees in group I (Carl and Japie) were immunized by five intravenous injections of $5 \times 10^{6}$ gamma-irradiated sporozoites at day 0 and weeks 8, 24, 44 and 65, and received three challenges at weeks 97 and 123 (Table 2, challenge doses). One year after the three challenges reported here, these chimpanzees were re-immunized once, and received one low-dose challenge and one high-dose challenge, which showed the same pattern of protection (data not shown; J.A.M.L. et al., manuscript in preparation). In group II, Gerda received NR2 lipopeptide at day 0 and weeks 3,13 and 31 as described ${ }^{19}$. She was then boosted with the mixture of LSA-3 GST-rec at weeks 40, 45, 48 and 50. Control chimpanzee Lianne received Montanide ISA51 alone. Challenges were done at week 60 . Chimpanzees in group III were immunized at day 0 and weeks 3 and 6. Mopia and Mgbado received LSA-3 as a mixture of NR1, NR2 and CT1 lipopeptides and of RE peptide, whereas Judy was injected with LSA-3 GST-rec adsorbed to latex microbeads. Low-dose and high-dose challenges were done at weeks 21 and 29, respectively. In group IV, Patty received LSA-3 (lipo)peptides, but without lipopeptide CT1, whereas Wendy and Willy were injected with LSA-3 GST-rec emulsified in SBAS2 (SmithKline Beecham Biologicals, Rixensart, Belgium), whose efficacy has been recently established in humans ${ }^{4,5}$. Control chimpanzee Helen received SBAS2 only. All chimpanzees were immunized at weeks 0,4 and 8 and were challenged with $2 \times 10^{4}$ sporozoites at week 13 . In group V, Cindy and Marty were both immunized at weeks $0,4,8$ and 26 with LSA-3 GST-rec in SBAS2 (as in group IV). Emmanuel and Fauzi were immunized at weeks 5, 8, 11 and 26 with 18 krad- and 30 krad-irradiated sporozoites, respectively, as were Carl and Japie (group I). Fauzi was considered the negative control and Emmanuel, the positive control for this group. Low-dose and high-dose challenges were done at weeks 33 and 46, respectively, in all four chimpanzees.

NF54 sporozoites were obtained from dissected salivary glands of infected Anopheles gambiae as described ${ }^{30}$. Sporozoites were pooled, resuspended in PBS and injected intravenously. All chimpanzees in each group were challenged with the same pool of sporozoites. Because of the cost, extensive evaluation of the minimal infective dose has not been undertaken; however, challenge with $5 \times 10^{3}$ sporozoites, the lowest dose used so far, has proven infective in four other chimpanzees (A.W.T., unpublished data).

Determination of the protective status. For groups I, II, IV and V, chimpanzee blood was obtained on days 5-10, evaluated by thick- and thin-film Giemsa-stained preparations, and confirmed in all cases by in vitro culture (data not shown), as described ${ }^{24}$. For chimpanzees in group III, blood obtained every day on days 5-18, then every other day up to day 30 , was used to prepare thin and thick smears, which were Giemsa-stained and examined by two separate microscopists. A chimpanzee was considered totally protected when no parasites could be detected in the circulation blood, by direct microscopy and by long-term culture, or partially protected when time to patency was delayed by 1 or more days compared with that in control chimpanzees. In mice, these delays correspond to a protection of $80 \%$ ( $24 \mathrm{~h}$ ) or $96 \%$ ( $48 \mathrm{~h}$ ) against sporozoite challenges. In humans, a 12-hour delay was calculated to correspond to a $92 \%$ reduction of liver forms after sporozoite challenges ${ }^{24}$. In a limited number of chimpanzees, a liver biopsy was done by a veterinary doctor on anesthetized chimpanzees on day 5 after a high-dose challenge. Material was fixed, and sections $4 \mu \mathrm{m}$ in thickness were made and stained by Giemsa-collophonium ${ }^{31}$ before the liver forms in 300 sections (average area, $0.8 \mathrm{~cm}^{2}$ ) were counted microscopically. All chimpanzees were treated with chloroquine immediately after the period of observation regardless of their protective status.

\section{Acknowledgments}

We thank G. Snounou for advice and review of the manuscript, G. Langsley for the gift of the K1 P. falciparum genomic library, D. Haynes and R. Edelman for the gift of sera from human volunteers, A. Asavanich for her contribution to sporozoite studies, and C. Blanc, S. Nègre, T. Kaidoh and J. Wubben for technical support. This work was supported by the Science and Technology for Development Programme of the Commission of the European Communities, grants CT 95-0016 and 98-0387.

\section{RECEIVED 7 AUGUST; ACCEPTED 3 OCTOBER 2000}

1. Herrington, D. et al. Successful immunization of humans with irradiated malaria sporozoites: humoral and cellular responses of the protected vaccinees. Am. J. Trop. Med. Hyg. 45, 539-547 (1991). 
2. Egan, J.E. et al. Humoral immune responses in volunteers immunized with irradiated Plasmodium falciparum sporozoites. Am. J. Trop. Med. Hyg. 49, 166-173 (1993).

3. Facer, C.A. \& Tanner, M. Clinical trials of malaria vaccines: progress and prospects. Adv. Parasitol. 39, 1-68 (1997)

4. Stoute, J.A. et al. A preliminary evaluation of a recombinant circumsporozoite protein vaccine against Plasmodium falciparum malaria. New Engl. J. M ed. 336, 86-91 (1997).

5. Stoute, J.A. et al. Long-term efficacy and immune responses following immunization with the RTS, S malaria vaccine. J. Infect. Dis. 178, 1139-1144 (1998)

6. Mellouk, S., Lunel, F., Sedegah, M., Beaudoin, R.L. \& Druilhe, P. Protection against malaria induced by irradiated sporozoites. Lancet 335, 721 (1990).

7. Scheller, L.F. \& Azad, A.F. Maintenance of protective immunity against malaria by persistent hepatic parasites derived from irradiated sporozoites. Proc. Natl. Acad. Sci. U. S. A. 92, 4066-4068 (1995).

8. Druilhe, P., Rénia, L. \& Fidock, D. Immunity to liver stages. in M alaria. Parasite biology, pathogenesis and protection (ed. Sherman, I.W.) 513-543 (American Society for Microbiology, Washington D.C., 1998).

9. Marchand, C. \& Druilhe, P. How to select P. falciparum pre-erythrocytic antigens in an expression library without defined probe in WHO Bulletin 68, 158-164 (1990).

10. Guerin-Marchand, C. et al. A liver stage-specific antigen of Plasmodium falciparum characterized by gene cloning. Nature 329, 164-167 (1987)

11. Fidock, D.A. et al. Plasmodium falciparum liver stage-specific antigen-1 is well conserved and contains potent B and T cell determinants. J. Immunol. 153, 190-204 (1994).

12. Bottius, E. et al. A novel Plasmodium falciparum sporozoite and liver stage antigen (salsa) defines major B, T helper, and CTL epitopes. J. Immunol. 156, 2874-2884 (1996).

13. Kemp, D.J., Cowman, A.F. \& Walliker, D. Genetic diversity in Plasmodium falciparum. Adv. Parasitol. 29, 75-149 (1990).

14. Gardner, M.J. et al. Chromosome 2 sequence of the human malaria parasite Plasmodium falciparum. Science 282, 1126-1132 (1998).

15. Moelans, I.I.M.D. \& Schoenmakers, J.G.G. Crossreactive antigens between life cycle stages of Plasmodium falciparum. Parasitol. Today 8, 118-123 (1992).

16. Barnes, D.A., Wollish, W., Nelson, R.G., Leech, J.H. \& Petersen, C. Plasmodium falciparum: D260, an intraerythrocytic parasite protein, is a member of the glutamic acid dipeptide-repeat family of proteins. Exp. Parasitol. 81, 79-89 (1995).

17. Kwiatkowski, D. \& Marsh, K. Development of a malaria vaccine. Lancet $\mathbf{3 5 0}$ 1696-1701 (1997)

18. Anders, R.F. \& Saul, A. Malaria Vaccines. Parasitol. Today 16, 444-447 (2000).

19. Ben Mohamed, L. et al. Lipopeptide immunization without adjuvant induces potent and long-lasting B, T helper, and cytotoxic T lymphocyte responses against a malaria liver stage antigen in mice and chimpanzees. Eur. J. Immunol. 27, 1242-1253 (1997).

20. Benmohamed, L. et al. High immunogenicity in chimpanzees of peptides and lipopeptides derived from four new Plasmodium falciparum pre-erythrocytic molecules. Vaccine 18, 2843-2855 (2000).

21. Aidoo, M. et al. CTL epitopes for HLA-B53 and other HLA types in the malaria vaccine candidate liver stage antigen-3. Infect. Immun. 68, 227-232 (2000).

22. Miyamoto, M.M., Koop, B.F., Slightom, J.L., Goodman, M. \& Tennant, M.R. Molecular systematics of higher primates: genealogical relations and classification. Proc. Nat. Acad. Sci. U.S.A. 85, 7627-7631 (1988).

23. Nussler, A.K. et al. In vivo induction of the nitric oxide pathway in hepatocytes after injection with irradiated malaria sporozoites, malaria blood parasites or adjuvants. Eur. J. Immunol. 23, 882-887 (1993).

24. Murphy, J.R., Baqar, S., Davis, J.R., Herrington, D.A. \& Clyde, D.F. Evidence for a 6.5-day minimum exoerythrocytic cycle for Plasmodium falciparum in humans and confirmation that immunization with a synthetic peptide representative of a region of the Circumsporozoite protein retards infection. J. Clin. Microb. 27, 1434-1437 (1989).

25. Gahery-Segard, H. et al. Multiepitopic B- and T-cell responses induced in humans by a Human Immunodeficiency Virus type 1 lipopeptide vaccine. J. Virol. 74, 1694-1703 (2000).

26. Bourgault, I. et al. Simian immunodefisciency virus as a model for vaccination against HIV: Induction in rhesus macaques of GAG or NEF specific cytotoxic T lymphocytes by lipopeptides. J. Immunol. 152, 2530-2537 (1994).

27. Vitiello, A. et al. Development of a Lipopeptide-based Therapeutic vaccine to treat Chronic HBV Infection. Induction of a primary Cytotoxic T Lymphocyte response in Humans. J. Clin. Invest. 95, 341-349 (1995).

28. Londono, J.A., Gras-Masse, H., Dubeaux, C., Tartar, A. \& Druilhe, P. Secondary structure and immunogenicity of hybrid synthetic peptides derived from two Plasmodium falciparum pre-erythrocytic antigens. J. Immunol. 145, 1557-1563 (1990).

29. Roggero, M.A. et al. Synthesis and immunological characterization of 104-mer and 102-mer peptides corresponding to the $\mathrm{N}$ - and C-terminal regions of the Plasmodium falciparum CS protein. M olec. Immunol. 32, 1301-1309 (1996).

30. Ponnudurai, T. et al. Sporozoite load of mosquitoes infected with Plasmodium falciparum. Trans. R. Soc. Trop. Med. Hyg. 83, 67-70 (1989).

31. Druilhe, P., Puebla, R.M., Miltgen, F., Perrin, L. \& Gentilini, M. Species- and stage specific antigens in exoerythrocytic stages of Plasmodium falciparum. Am. J. Trop. Med. Hyg. 33, 336-341 (1984).

32. Meis, J.F.G.M. et al. Plasmodium falciparum: studies on mature exoerythrocytic forms in the liver of the chimpanzee, Pan troglodytes. Exp. Parasitol. 70, 1-11 (1990). 